\title{
Data-driven regionalization for analyzing the spatiotemporal characteristic of air quality in China
}

\author{
Sheng $\mathrm{Li}^{\mathrm{a}}{ }_{*}$, Jiangtao $\mathrm{Liu}^{\mathrm{a}}$, Chao $\mathrm{Wu}^{\mathrm{b}, \mathrm{c}}$ \\ ${ }^{a}$ Shenzhen Municipal Planning \& Land Real Estate Information Centre, Shenzhen 518034, China, Sheng Li Shenglee@whu.edu.cn \\ ${ }^{b}$ School of Geographic and Biologic Information, Nanjing University of Posts and Telecommunications, Nanjing 210023, China. \\ ${ }^{c}$ Smart Health Big Data Analysis and Location Services Engineering Lab of Jiangsu Province, Nanjing University of Posts and \\ Telecommunications, Nanjing 210023, China. \\ * Corresponding author
}

Keywords: Air Quality, GWPCA, Spatial Clustering, Spatiotemporal Analysis, China

\begin{abstract}
:
With the development of urbanization and industrialization, the degradation of ambient air quality has become a serious is sue that impacts human health and the environment; thus, it has attracted more attention from scholars. Usually, the mass concentrations of sulfur dioxide (SO2), nitrogen dioxide (NO2), carbon monoxide (CO), ozone (O3) and particulate matter with an aerodynamic diameter less than $10 \mu \mathrm{m}$ and $2.5 \mu \mathrm{m}$ (PM 10 and PM2.5) are used to evaluate air quality. A commonly used data-driven regionalization framework for studying air quality issues, identifying areas with similar air pollution behavior and locating emission sources involves an incorporation of the principal component analysis (PCA) with cluster analysis (CA) methods. However, the tradition al PCA does not consider spatial variations, which is a notable issue in geographic studies. This article focuses on extracting the local principal components (PCs) of air quality indicators based on a geographically weighted principal component analysis (GWPCA), which is superior to the PCA when considering spatial heterogeneity. Then, a spatial cluster analysis (SCA) is used to identify the areas with similar air pollution behavior based on the results of the GWPCA. The results are all visualized and show that the GW PCA has a higher explanatory ability than the traditional PCA. Our modified frame work based on the GWPCA and SCA for assessing air quality can effectively guide environmentalists and geographers in evaluating and improving air quality from a new perspective. Furthermore, the visualization results can be used by city planners and the government for monitoring and managing air pollution. Finally, policy suggestions are recommended for mitigating air pollution via regional collaboration.
\end{abstract}

